\title{
Proceso de lectoescritura en los adolescentes de secundaria de la comunidad de Ayahualulco
}

\author{
Kenneth Joely Gómez Vázquez ${ }^{1}$ \\ Fiordalizo Maldonado Vargas ${ }^{2}$ \\ Paulina Morales Haro ${ }^{3}$ \\ Rosa María Romero Martínez ${ }^{4}$
}
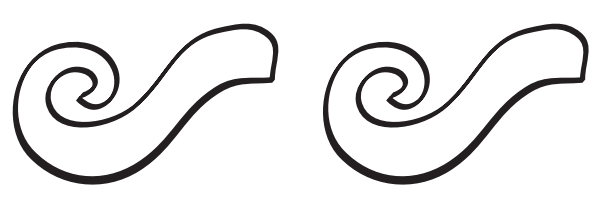

\section{Resumen}

La presente investigación analiza el proceso de lectoescritura en alumnos que asisten a la secundaria de La Salle, en la comunidad rural de Ayahualulco, Veracruz, a través de un proceso de investigación de tipo cualitativo, in situ, y teniendo como instrumentos de recolección de datos las historias de vida, entrevistas y observaciones.

En términos generales, el análisis de datos indica que el proceso de lectoescritura se encuentra en un nivel medio, ya que se observó que hay un cierto acercamiento a la lectura por parte de los adolescentes y existe también una comprensión lectora. Los factores que están deficientes son la apreciación lectora, es decir, la expresión de los sentimientos y enseñanzas que la lectura les dejó; así como también, una notable deficiencia en la ortografía. Se constató que la familia es un factor que afecta a los alumnos para no tener el hábitos lectores, ya que a los padres no les interesan los libros; incluso algunos padres son analfabetas.

\begin{abstract}
The present investigation analyze the reading-writing process on secondary students from the La Salle school, in the community of Ayahualulco, Veracruz, through a process of qualitative investigation, in situ, and having as collecting instruments life stories, interviews and observations.
\end{abstract}

Generally, the analyze indicates that the reading-writing process is in a medium level, because it was observed that there is a certain kind of approach to the reading from the teenagers and, there is also, a reading comprehension, the deficient factors which are present are the reading appreciation, in other words, their feeling expression and teachings that the reading left to them; also there is a notorious deficient at the spelling. It was noticed that the family is a factor that affects to the students to don't have the reading habit, because the parents are not very interested into the reading, or, in some cases the parents are illiterate.

\section{Introducción}

\subsection{Planteamiento del problema}

El aprecio hacia la lectura es un hábito que generalmente no tienen los países cuyo nivel de desarrollo y el nivel educativo son bajos, tal es el caso de México. Se estima que en este país el promedio nacional de libros que lee una persona es de 2.9 por año de acuerdo a la Encuesta Nacional de Hábitos, Prácticas y Consumo Culturales, realizada en 2010 a 32,000 personas del país, aplicada por CONACULTA.

La misma encuesta reveló los porcentajes detallados del número de libros que lee un mexicano al año: $16.7 \%$ leen 1 libro, el 14.2\% leen 2 libros, el 16.7\% leen de 3 a 5 libros, el 9.1\% leen de 6 a 10 libros, un $4.2 \%$ leen 11 o más libros, y un $33.5 \%$ no lee ningún libro. Las cifras son alarmantes.

1 Octavo semestre de la Licenciatura en Ciencias de la Educación. (kenny_gv@hotmail.com) 2 Octavo semestre de la Licenciatura en Ciencias de la Educación. (catalin_mv@hotmail.com) 3 Octavo semestre de la Licenciatura en Ciencias de la Educación. (moralesharo_pqm@hotmail.com) 4 Octavo semestre de la Licenciatura en Ciencias de la Educación. (rositafresita0991@hotmail.com) 


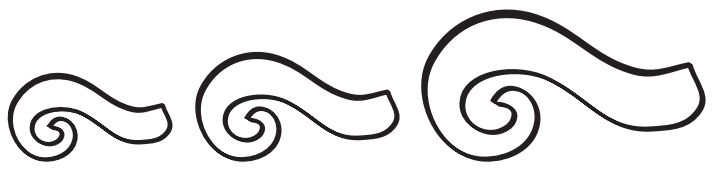

Hoy en día se vive lo que se ha denominado "La era del conocimiento", esto significa que el poder se encuentra en las manos de aquellos que no sólo tienen información a manos llenas, sino que la utilizan en su vida diaria, saben aplicarla y saben en dónde buscarla; pero sobre todo, saben comprenderla.

La globalización ha sido en gran parte la responsable de que vivamos en esta era. El problema es que esta información no está disponible para todos, sino que sólo para unos cuantos. De acuerdo con un informe presentado por Alicia Bárcena, secretaria ejecutiva de la CEPAL en 2011, en América Latina viven aproximadamente 174 millones de personas en situación de pobreza y 73 millones de ellos viven en condiciones de pobreza extrema o indigencia. Se estimó que en el 2011 la tasa de pobreza disminuyó a 30,4\% de la población, mientras que la de indigencia ascendió a 12,8 $\%$, debido a que el alza en los precios de los alimentos contrarrestaron el incremento en los ingresos de los hogares.

Mientras en países como Perú, Ecuador, Argentina, Uruguay y Colombia la pobreza se ha reducido significativamente, en México las tasas de pobreza aumentaron, aproximadamente a 1.5 puntos porcentuales, junto con Honduras.

La pobreza y la falta de educación siempre han ido de la mano como los problemas más difíciles por enfrentar por los gobiernos de países como México. Un país que carece de recursos económicos, carecerá de educación y viceversa. Un problema lleva al otro. Un país que carece de educación carece de todo lo demás.

La lectura, o mejor dicho el hábito de la lectura, se convierte en una problemática en México a causa del escaso número de personas que comprenden lo que leen. Si la educación es una herramienta que puede sacar a un país de la pobreza, si no se lee, entonces, ¿cómo se logrará dicha meta? La escuela es un elemento

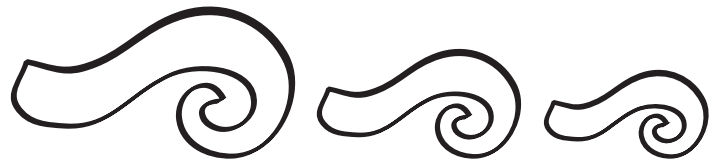

clave que puede lograr que México comience a disminuir sus índices de pobreza, en lugar de aumentarlos. ¿De qué manera? logrando una escuela de calidad, tanto en sus procesos como en sus resultados.

Otra variable dentro de la problemática es el proceso de lectoescritura. Este proceso se puede comprender mejor al desarticularlo y entender que la lectura y la escritura son sistemas que van de la mano, pero que cada uno se adquiere de manera y en momentos diferentes.

La lectura se compone de tres sistemas, uno simbólico visual, otro simbólico auditivo y por último uno semántico. Puede decirse que "el aprendizaje de la lectura constituye una relación entre lo que se oye y se dice con lo que se ve y lee." (Da Fonseca, 2010, p.120).

Además la lectura involucra una serie de pasos, que los niños que se encuentran en el proceso de adquirirla, deben llevar a cabo. Entre los pasos se ven involucrados tanto el sentido de la vista, como el sentido del oído, incluso el tacto; el niño debe hace una integración de los símbolos y de los sonidos y por último comprender que esa combinación tiene un significado (Da Fonseca, 2010, p.121).

La escritura, por otro lado, es un proceso que tiene lugar, una vez que la lectura se ha consolidado y que le lleva más tiempo adquirir al ser humano. Para que se dé la escritura la persona debe tener una intención o voluntad, después una elaboración ordenada de sus ideas, prosiguiendo con la remembranza de las grafías que utilizará y el orden en que deben ir, por último se da la escritura (Da Fonseca, 2010, p. 125).

Como se puede observar, ambos procesos son complejos y si la persona que se encuentra en el proceso de adquirirlos no está siendo guiada correctamente, tendrá deficiencias que lo llevarán a presentar dificultades más adelante y posiblemente su gusto por la lectura y la escritura resultará escaso. 


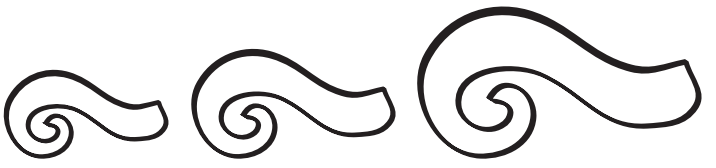

Se deben revisar a fondo los procesos que se llevan a cabo en las escuelas de México, específicamente en lo concerniente al proceso de lectoescritura y en comunidades donde la pobreza se agudiza, para que de este modo, se logren descifrar muchas interrogantes y se encuentre la raíz del problema.

\subsection{Objetivo General}

Analizar cómo se lleva a cabo la enseñanza y aprendizaje del proceso de lectoescritura en los jóvenes de secundaria de La Salle de Ayahualulco.

\subsection{Objetivos Específicos}

- Elegir la muestra de alumnos de secundaria de La Salle que se encuentra en la comunidad.

- Analizar los factores que influyen en el proceso de lectoescritura de los jóvenes, tales como el maestro, el alumno mismo y el ambiente educativo.

- Enlistar las debilidades y fortalezas encontradas.

- Diseñar un plan de trabajo sugerente en el que se atiendan las debilidades y se potencialicen las fortalezas en los procesos lectores de los alumnos de secundaria.

- Presentar los resultados.

\subsection{Preguntas de investigación}

1. ¿Cuáles son las causas que influyen en el desempeño de la lectura en los alumnos de secundaria de La Salle, en la comunidad de Ayahualulco?

2. ¿Cuáles son las consecuencias de las debilidades en el hábito de la lectura en los alumnos de secundaria de La Salle, en la comunidad de Ayahualulco?

3. ¿Cuáles son las debilidades en el hábito de la lectura de los alumnos de secundaria de La Salle, en la comunidad de Ayahualulco?

4. ¿Cuáles son las fortalezas en el hábito de la lectura de los alumnos de secundaria de La Salle, en la comunidad de Ayahualulco?

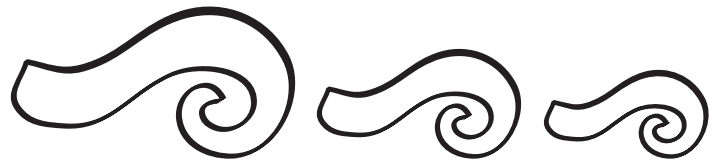

5. ¿Qué estrategias se pueden implementar para mejorar el hábito de la lectura en los alumnos de secundaria de La Salle, en la comunidad de Ayahualulco?

\subsection{Justificación:}

Por los motivos mencionados anteriormente es que se decidió realizar la investigación.

Al igual que todos los proyectos que se llevan a cabo en la Universidad La Salle, la investigación se basa en las urgencias de PERLA (Proyecto Educativo Regional Lasallista Latinoamericano).

Las urgencias educativas en las que se ubica el proyecto son:

1.3 Educación de Calidad: porque se pretende elevar el nivel de los alumnos para que cuenten con las mismas oportunidades que un niño que cursa el mismo grado en la ciudad, se trata de equilibrar las condiciones de aprendizaje de los alumnos para que puedan tener un mejor futuro.

\subsection{Supuesto:}

Los jóvenes de secundaria de la comunidad de Ayahualulco presentan deficiencia en el proceso de lectoescritura, debido a la falta de recursos didácticos para estimular el proceso de enseñanza aprendizaje.

\section{Marco Teórico}

\subsection{Marco Histórico}

Antes de explicar el concepto de lectoescritura es importante saber de dónde proviene, cuál es su origen y quienes fueron los primeros que lo estudiaron.

Durante la época de la colonia hubo una gran preocupación por evangelizar al pueblo de la Nueva España. Durante los siglos XV, XVI, XVII se fundaron escuelas muy importantes 


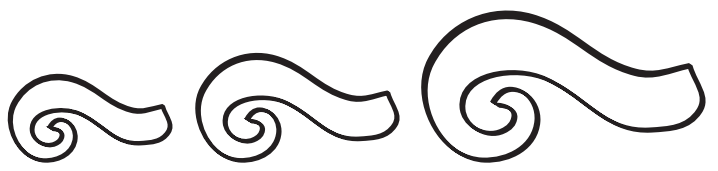

como El Colegio de Tlatelolco, el de San Nicolás de Pátzcuaro y la Real Universidad Pontificia (Cantón, 2009, p.3). En aquellos días la educación tenía un papel específico como hoy día, pero la prioridad no era enseñar a leer y escribir a todo el pueblo, como la mayoría de nosotros sabe, la educación estaba solo al alcance de la gente adinerada o acaudalada, pero fue a partir de que se aprobó una ley en la Constitución de Cádiz que establecía que solo podrían votar quienes supieran leer y escribir, ahí fue que tomó importancia el proceso de lectoescritura, este suceso hizo que la preocupación y el objetivo de la escuela cambiara (Cantón, 2009, p.8).

A partir de ese día la gente que se dedicaba a enseñar a los otros se dio a la tarea de enseñar a las personas que no sabían leer ni escribir.

El Tercer Congreso Nacional de Educación Primaria se llevó a cabo en septiembre de 1910. A este congreso asistieron dos personas que tuvieran un grado elevado en educación primaria por cada uno de los diferentes estados. El congreso estaba dirigido a maestros y consistía en darles una visión de lo que era la educación nacional, ya que la ley no se estaba cumpliendo; se convocó a los maestros a unir sus esfuerzos para lograr los objetivos que se habían planteado en los congresos anteriores y que aún no se cumplían, fue en este encuentro cuando se dieron a conocer las experiencias de los maestros que se quejaban de una forma trágica, ya que la situación del maestro era precaria, en ese momento se pensaron posibles soluciones para mejorar la situación de los maestros, se propuso que los maestros se reunieran de forma periódica en asambleas para agilizar el proceso educativo. En este congreso se dieron a conocer los alcances que tenía la educación en cada uno de los estados (Bazant, 2006).

Conforme pasó el tiempo el proceso de la lectoescritura fue tomando mayor importancia y diversos teóricos reconocidos empezaron a

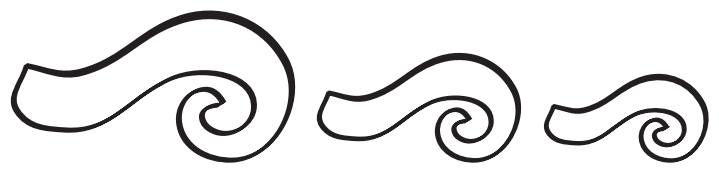

preguntarse ¿Cómo es que los niños adquieren y se apropian del lenguaje escrito y oral? Y surgieron diversas teorías de distintos autores como la teoría sociocultural de Lev Vigotsky (1885-1934) en la cual la premisa base era que el niño aprende a partir de las interacciones que tiene con los otros, en conjunto con la sociedad; la teoría cognitiva de Jean Piaget (18961981) que establece estadios de desarrollo a través de los cuales el niño va madurando física y cognitivamente, aunque tiene importancia muchos no toman al $100 \%$ su teoría ya que para hacerla se basó en el desarrollo de sus hijos (Chávez, 2001).

Las investigaciones que surgieron posteriormente se basaron en las teorías de los autores mencionados líneas arriba, a continuación se mencionarán dichas teorías:

Ferreiro (1983-1994) dice que en el proceso de aprendizaje de la lectoescritura interviene en gran medida el contexto sociocultural y la función social que tiene la lengua escrita para comunicar significados ya que por medio de ésta se trasmite lo que la persona piensa, cree, conoce y siente. Y tomando como base este planteamiento M.A.KHalliday (1986) identificó seis categorías de funciones lingüísticas que se desarrollan dentro del contexto social: 1.regulatoria, 2. interaccional, 3. personal, 4. imaginativa, 5 . lenguaje heurístico, 6 . lenguaje informativo (Chávez, 2001). De acuerdo con esta teoría en el proceso de lectoescritura interviene en gran medida el contexto sociocultural y la función que tiene la lengua escrita para dar significados, ya que es a través de ésta que el individuo puede transmitir lo que piensa, siente y necesita.

Las investigaciones de Norman Jackson, 1982, Chomsky, 1971; Clay, 1975; Cohn, 1981 citadas por Ruiz, 1996 sobre los lectores naturales, es decir niños y niñas que aprenden a leer en su hogar y sin instrucción formal, coinciden en que el lenguaje emerge de una necesidad de comunicarse con los demás y que el ambiente 


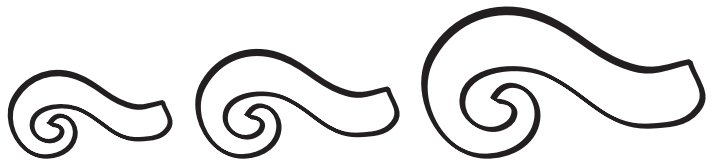

sociocultural ejerce una gran influencia en el desarrollo de esta habilidad. El término de lectores naturales se aplica a los niños que se desarrollan en ambientes que se caracterizan por tener acceso a todo tipo de materiales para la lectura y escritura, aquí estas actividades se ven de forma cotidiana; los niños interactúan poco a poco con ellos, lo hacen movidos por la curiosidad y son ellos los que empiezan por iniciativa propia a descubrir la lectoescritura.

\subsection{Marco Conceptual}

En la actualidad se habla constantemente de fomentar una cultura adecuada para impulsar el hábito de la lectura en los niños y niñas de México, para que así se pueda implementar un proceso de lectoescritura más eficiente, es por ello que en esta investigación nos centraremos en estos dos factores.

Para ello, primero es necesario revisar y dar significado a éstos para así entender sobre qué hablaremos a lo largo de nuestra investigación.

- Lectoescritura

- Una definición de lectoescritura bajo tres etapas es: "La primera es una conceptualización de la lectoescritura como herramienta. La segunda es la adquisición de la lectoescritura, es decir, el aprendizaje de habilidades para la lectura y la escritura. La tercera es la aplicación práctica de estas actividades de manera significativa para el que aprende. Cada etapa depende de la anterior, cada una es un componente necesario para la lectoescritura" (Harman, 1970, p 228 citado por Lorenzo, 1994).

- Responde a la visión vigente del niño, quien construye su conocimiento socialmente; y del aprendizaje, que ocurre en contextos sociales. No es un nombre nuevo para la misma cosa; representa la relación indisoluble entre ambos procesos (Ruiz, 2007 p.1).

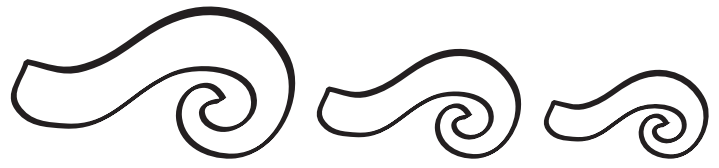

Las definiciones que acabamos de revisar nos sirvieron para deducir las siguientes conclusiones:

La lectoescritura es un proceso de enseñanza aprendizaje que es esencial en los años de la educación primaria, hace alusión a la capacidad de leer y escribir del niño, representa la relación entre ambas y la importancia de las mismas de que vayan de la mano en el desarrollo adecuado del infante.

- Comprensión lectora:

- Es un procedimiento que toma en cuenta una serie de estrategias y técnicas que hay que saber utilizar de manera adecuada para construir el significado que cada texto propone (Zabala et al, 2000, p. 143).

- Es la forma en que el sujeto se relaciona con la lengua escrita. Implica que la persona esté familiarizada con los grafonemas, los signos de puntuación y otros signos auxiliares como los asteriscos, los guiones o los paréntesis y su aporte a la claridad de la información. Es también, la apropiación de los significados de un texto mediante el uso del contexto, la predicción, la recapitulación y la jerarquización de la información (webdianoia.com, 2006).

- Se puede entender como la capacidad para extraer sentido de un texto escrito.

Por lo tanto, después de analizar estas definiciones entendemos por comprensión lectora al proceso de aprendizaje que realiza el ser humano para entender un texto, para realizar una correcta reinterpretación de una lectura necesita estar familiarizado con los signos de puntuación y otros signos del idioma así como el nivel de complejidad de las palabras que el texto requiera para ser comprendido e interpretado adecuadamente.

- Hábito de lectura:

- Los hábitos se forman mediante las repeticiones uniformes y frecuentes, que son acompañadas por un conocimiento de satisfacción producido (Kelly, 1982, p. 175). 


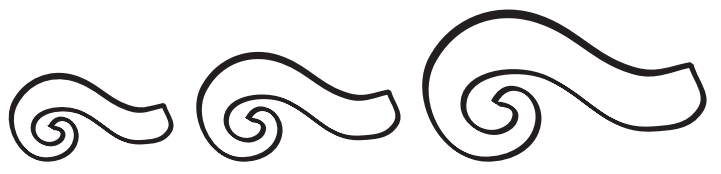

- La lectura es una actividad lingüístico-cognitiva que tiene como objetivo la comprensión del lenguaje escrito; en el cual el lector lleva a cabo la construcción de su propio significado. (Álvarez, 2004 p. 9).

Por lo tanto, se entiende por hábito de lectura la manera de actuar, adquirida con la práctica, de leer constantemente para la comprensión de un texto y construcción de un significado propio.

Después de haber revisado algunos puntos de vista sobre los temas que trataremos durante la investigación, continuamos con el análisis del marco teórico de la investigación.

\subsection{Marco teórico}

Para fines de esta investigación, la postura desde la cual se mirará al problema será el enfoque del lenguaje integral, propuesto por los autores Kenneth y Yetta Goodman, en 1992.

La postura de los autores expresa que:

El aprendizaje de la lengua escrita es un aprendizaje natural. Cualquier niño aprende a hablar sin que se le enseñe explícitamente hacerlo (...) porque está rodeado de personas que usan su lengua para comunicarse. Asimismo, el niño que vive en un medio social que usa la escritura como medio de comunicación aprenderá a leer y escribir porque quiere y necesita participar de las convencionalidades de su medio, porque necesita comunicarse (Goodman, 1992 en Vernon, 2011).

Esta postura desde la que se concibe al proceso de lectoescritura está basada en la teoría de Lev Vygotsky.

Vygotsky, psicólogo de origen ruso, se basaba en las relaciones sociales que todo ser humano establece desde que es pequeño, su historia, su cultura y sus funciones mentales superiores. Su teoría fue llamada histórico-cultural (Ivich, 1987).

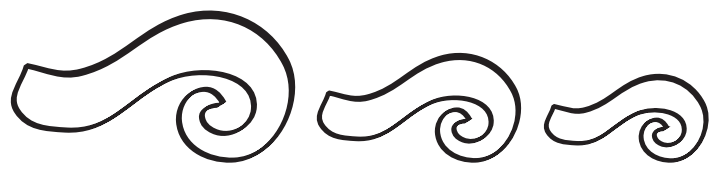

La tesis principal de Vygotsky es que "el ser humano se caracteriza por una sociabilidad primaria" (Ivich, 1987). Cuando Vygotsky expuso lo anterior, la comunidad científica y los psicólogos de aquella época no lo consideraron así y lo denominaron entonces un postulado. Hoy en día se conoce que la sociabilidad primaria es una tesis y puede afirmarse que es determinada genéticamente, que es un acto que se da de manera natural desde la primera infancia.

Dentro del ámbito de la enseñanza de la lectura, se encontró que existen nueve métodos utilizados, de los cuáles, el método que se quiere tomar también como parte de este marco teórico es el método ecléctico (Freeman, 1989). El método de enseñanza de la lectura "eclécti-

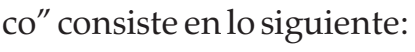

Puede ser llamado más apropiadamente método mixto (...). Se dan a los alumnos ejercicios preparatorios para promover habilidades en organización espacial, coordinación visomotora, discriminación auditiva, atención, memoria, y lenguaje oral como lo sugiere el método ideovisual. Luego se presentan los sonidos de las letras y los alumnos son estimulados a aprender el sonido, el nombre de la letra y el símbolo escrito para producir las letras. También se les enseña a tomar dictados, a copiar, a crear nuevas palabras, a visualizar la forma de las letras, a identificar los sonidos representados por las letras, a escribir las formas de las letras, y a entender la relación oralescrita de los materiales primarios (Peña y Verner, 1981, en Freeman, 1989).

La razón por la que se escoge a este método es porque parece ser un método que abarca todas las habilidades y características que un alumno debe desarrollar al aprender a leer.

Parece un método completo, incluyente e integral. Lo que se pretende, al tener este método como referente teórico es compararlo con el método que utilizan los niños en la primaria de Ayahualulco y analizar las fortalezas y debilidades que pueda tener. 


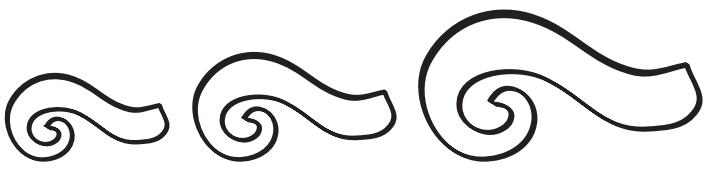

\subsection{Marco referencial}

La lectoescritura es una base fundamental para tener una comprensión no sólo de libros sino de todo el medio en general, por ejemplo que comprenda lo que habla y de lo que está hablando, además de que comprenda el mensaje de cualquier publicación. Aunque actualmente existe un problema en la enseñanza de esta habilidad.

En una investigación que se realizó en la Institución Educativa Rural San Pío X Sede Nariño, se demostró que la metodología de enseñanza sobre la lectoescritura es la que provoca bajo rendimiento académico y además se genera una actitud negativa por parte de los alumnos para aprender la lectura y la escritura. Algo más que influye en esto es que los padres de familia no apoyan a sus hijos en el aprendizaje sobre la lectoescritura, ya que ellos sólo lo dejan como un trabajo para la escuela y no lo ven cómo que es algo importante para que los niños tengan una mejor comprensión e interacción social (Marin, O. Zabala, R. Giraldo, S. Cardona, G., 2008).

Estos fueron resultados de una investigación realizada mediante una recolección de datos (como entrevistas, encuestas tanto a padres como a alumnos) además de observaciones directas y talleres para evaluar a los niños (Marin, O. Zabala, R. Giraldo, S. Cardona, G., 2008).

En otra investigación realizada sobre el caso de tres escuelas primarias de Tuxtla Gutiérrez, Chiapas; se presentaron otras consecuencias que causan la falta de atención sobre la lectoescritura. Nos dice que un problema es que los niños presentan ausentismo y niveles de maduración poco suficientes, esto a consecuencia de que los niños entran a la primaria a una edad temprana, y hay otros niños que tienen problemas de distracción (López, M., Ramos, R., Mancilla, M. 2008).

La adquisición de esta habilidad no es sólo para comprender los textos, sino que es de

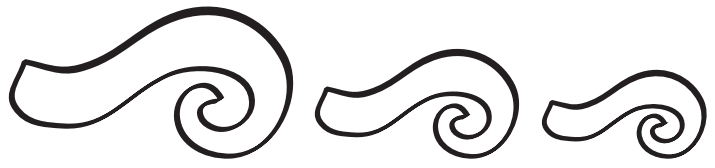

gran ayuda para comprender el medio. Es por ello que el desarrollo de la lectoescritura recae en el profesor, aunque él también debe saber que esto debe trabajarse con atención a la diversidad ya que no todos los niños aprenden de la misma manera ni al mismo ritmo. En esta investigación se realizó la prueba BENHALE (Batería evaluadora de las habilidades necesarias para la lectura y la Eescritura), en el cual se mostraron resultados de que los niños presentan problemas de coordinación viso-motora, con percepción y discriminación visual, problemas con el vocabulario, con la estructuración del espacio temporal y discriminación auditiva, entre otros(López, M. Ramos, R. Mancilla, M., 2008).

\section{Metodología}

\subsection{Enfoque}

La investigación que se realizará tendrá un enfoque cualitativo, ya que trataremos de describir las cualidades que tiene el problema de la lectoescritura en los jóvenes de secundaria de la comunidad de Ayahualulco.

La observación que se realizará con los jóvenes de secundaria será natural, no tendremos control sobre ellos solamente nos acercaremos para tener una convivencia con ellos y poder saber qué es lo que sucede en su casa, con sus amigos y en su vida con relación a los profesores. Es por ello que será llevado desde una perspectiva holística, ya que no sólo nos enfocaremos a su vida escolar, sino también a su vida personal y emocional. Todo esto, tal y como es una investigación cualitativa.

El método de la investigación es inductivo. Se harán observaciones de manera libre y natural, como antes se había mencionado, sin hacer prejuicios; todo ello con el fin de obtener conclusiones generales a través de situaciones particulares.

\subsection{Contexto}

La comunidad de Ayahualulco está localizada en la zona centro montañosa; limitando al 


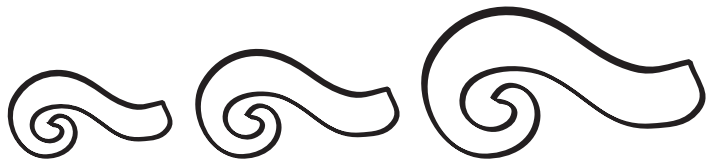

norte con Perote, al noreste con Xico, al este con Ixhuacán de los Reyes y al sur con el estado de Puebla. El municipio está ubicado en la parte más alta de la Sierra Madre Oriental.

Ayahualulco es denominada una comunidad rural, cuya principal actividad económica es la agricultura, principalmente del chayote.

El lugar donde se observó a los alumnos fue en el albergue de La Salle localizado en Ayahualulco. Los alumnos observados asisten ahí para estudiar la secundaria. Ellos viven ahí, como en una especie de internado, ya que sus hogares están en comunidades lejanas. Cada viernes se van a su casa y regresan los domingos por la tarde.

\subsection{Clasificación de la investigación}

Esta investigación cumplirá con las siguientes características: tendrá la finalidad de ser aplicada, porque buscará que los resultados obtenidos tengan una utilidad práctica dentro del contexto de la comunidad.

Además, la investigación también será de campo, porque la principal fuente de investigación serán las entrevistas, cuestionarios y observaciones naturales.

De acuerdo con el campo de estudio, la investigación será científica, porque se desarrollará a través del método científico. La investigación también será empírico-racional, porque busca ser reflexiva y sistemática., se relaciona con la característica de buscar la solución al problema de la investigación.

El método inductivo, porque pretende partir y analizar situaciones particulares de la problemática, para generalizar los resultados obtenidos, y así dar una respuesta general a la comunidad.

Por último, la investigación será colectiva porque los investigadores que integran el equipo son personas de un área de estudio igual.

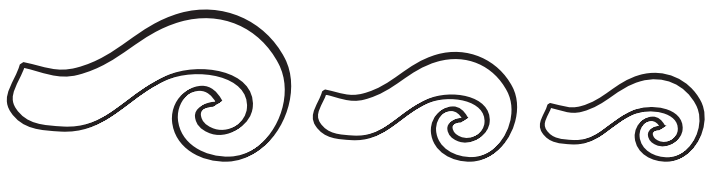

\subsection{Diseño de la investigación}

El diseño de esta investigación es de tipo no experimenta y transversal. La razón es porque el fenómeno a estudiar será respetado por lo que nuestra intervención como investigadores será baja, nos limitaremos a observarlo. Es transversal porque se compararán las variables de la problemática y se encontrará su relación.

El diseño de la muestra fue no probabilístico ya que la elección de los elementos no dependió de la probabilidad, sino, de las causas relacionadas con las características de la investigación como fueron la disponibilidad del número de alumnos, los alumnos ausentes etc. o de quien hizo la muestra ya que sólo se llevó un cierto número de instrumentos para evaluar y observar a los alumnos.

\subsection{Alcance de la investigación}

Para esta investigación, determinamos que tenemos un solo tipo de alcance: descriptivo. Esto porque tomando en cuenta la información obtenida y sus cualidades, desarrollaremos sus principales características y realizaremos la relación que existe con nuestro tema de estudio.

\subsection{Sujetos, universo y muestra.}

Nuestro universo es la comunidad de Ayahualulco y algunas comunidades aledañas a ésta. En algunas comunidades se pudieron visitar las escuelas y realizar ahí las entrevistas.

En la primaria Vicente Guerrero se realizaron, durante tres días, talleres sobre los procesos lectores de los alumnos, se atendieron a cuatro grupos.

Con respecto a los docentes, se les aplicaron seis encuestas. Participaron para contestarla, aunque al principio no mostraban interés por- 


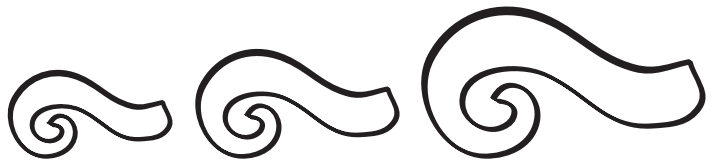

que creían que se les haría tarde para salir del trabajo; pero al final se obtuvo bastante información para saber cómo manejan las actividades de la lectura en esa primaria.

Al igual que en la primaria, a los niños y niñas del albergue de La Salle se les impartió un taller con tres módulos. Eran 37 alumnos los que estaban presentes durante el taller, pero sólo se eligieron a 30 niños para la aplicación de los instrumentos (historia de vida y observación).

A los alumnos de $5^{\circ}$ semestre de bachillerato se les impartió un taller de cuatro módulos sobre la comprensión lectora; eran 57 alumnos los que estaban presentes durante la realización de éstos. Se obtuvo un gran trabajo de su parte, ya que mostraban interés y participaban durante la realización, facilitándonos la labor para una buena aplicación de temas.

Un profesor de La Salle Ayahualulco colaboró con una encuesta, después de realizar la comida hubo acercamiento hacia él; y cooperó mucho con sus respuestas, esto nos ayudó bastante en el acercamiento con los docentes y en la obtención de los resultados de diferentes escuelas y ubicaciones.

\section{- LaMesa}

Solamente se realizó una encuesta en esta comunidad; ya que el maestro que estaba atendiendo era solo uno; la escuela que se visitó fue la Nicolás Bravo. Esperamos a que les pusiera un trabajo a sus alumnos para que nos regalara unos minutos de su tiempo y poder llevar a cabo la entrevista lo más rápido posible.

\section{- Chirinos}

En esta comunidad se logró visitar dos escuelas, se pudieron realizar tres encuestas a los docentes. Todos colaboraron, y nos recibieron con una buena actitud. Solo hubo un profesor que algunas veces no entendía las preguntas, pero eso no era problema ya que le explicába-

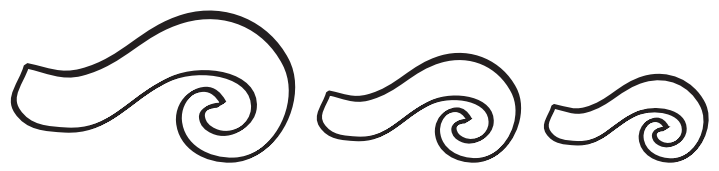

mos con otras palabras. La escuela visitada era de CONAFE, una de ellas tenía el nombre de Miguel Hidalgo, la otra escuela visitada fue el DIF.

\section{- La Manzanita}

En La Manzanita se visitó una escuela, donde se realizaron dos encuestas. Al igual que las otras instituciones, ésta también era una escuela multigrado; pero de igual manera hubo participación de parte de los docentes. Nos compartieron cómo es que ellos llevan a cabo prácticas sobre la lectura, y mencionaron que se hacen preguntas a los alumnos para saber si ellos comprendieron.

\section{- Cocalcingo}

La escuela que estaba en esta comunidad, era al igual que la comunidad de Chirinos. Sólo había un maestro atendiendo primaria, aunque sólo contaba con un salón, pudo dejarlos trabajando para poder responder a nuestra encuesta. Él menciono que trabaja la lectura con todos sus alumnos.

\subsection{Instrumentos de recolección}

Los instrumentos utilizados para esta investigación son: historias de vida, observación y entrevista.

La entrevista se elaboró con base en el tema del proceso de la lectura y estuvo dirigida a padres de familia y docentes de las diferentes comunidades visitadas. Las preguntas fueron diseñadas con el fin de obtener información referente al tiempo y forma que los padres y docentes tienen con sus hijos o alumnos.

La historia de vida fue diseñada para los alumnos de secundaria, lo que ellos debían contarnos era acerca del libro que más les haya significado en su vida y relatarnos cómo se sintieron y qué enseñanza les dejó. La observación fue diseñada para medir su comprensión lectora.

$$
\text { ๑ } 35 @
$$




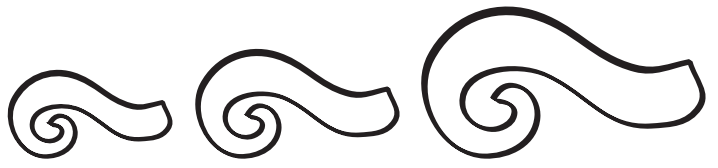

La historia de vida y la observación son los instrumentos elegidos para hacer la triangulación de datos. La observación fue previamente planeada y diseñada con los ítems predeterminados. Los ejemplos de los instrumentos se encuentran en el apartado de Anexos.

\subsection{Procedimiento}

La investigación cualitativa se llevó a cabo en la comunidad de Ayahualulco, Veracruz y en otras comunidades cercanas. Se realizaron 30 historias de vida, 25 entrevistas a los padres de familia y 14 entrevistas a docentes de diferentes comunidades.

Para llevar a cabo las entrevistas a los 25 padres de familia se visitaron comunidades como: La Manzanita, Rancho Nuevo, Coscomatepec, Chirinos y Ayahualulco. Al llegar a cualquier comunidad, se buscaban a los sujetos entrevistados; se mantenía una relación cercana, ya que se tenía que saludar y presentarse ante ellos para que así hubiera más de confianza. Las entrevistas les eran leídas y si no quedaba claro algún término se buscaba algún sinónimo, con el propósito de que a ellos les resultara fácil comprender y así contestaran con sinceridad. En una ocasión una de las personas entrevistadas tuvo un recuerdo triste por lo que le dio nostalgia, y cuando eso sucede el entrevistador debe mostrar un apoyo para que la persona quedara tal y como se encontró.

Con los padres de familia no hubo mucho problema para la recolección de datos, y es que en todas las comunidades; ellos mostraban interés por ayudar.

Las historias de vida y los datos de observación fueron aplicados a las mismas personas; se eligieron a 30 niños del albergue de La Salle Ayahualulco para que contestara cada uno de ellos, una historia de vida; dicha actividad les quedó como tarea para el día siguiente. En el

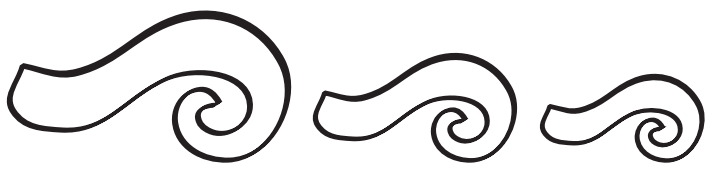

taller que se proporcionó el día miércoles 7 de noviembre de 2012 se recogió toda la información de las historias de vida para después aplicar las observaciones. Los chicos estuvieron en equipo por lo que cada integrante del equipo observó a 6 niños; se realizaron círculos de lectura, preguntas a mitad del texto y al finalizar conclusiones se aplicó el instrumento de observación.

Las entrevistas que se realizaron a los docentes fueron 14. Los maestros que participaron son de las siguientes instituciones: Nicolás Bravo de La mesa, La Salle de Ayahualulco, Miguel Hidalgo de Chirinos, DIF de Chirinos, Escuela CONAFE de la comunidad de Cocalcingo y la primaria Vicente Guerrero de Ayahualulco. Se entrevistaban a los docentes que tuvieran tiempo disponible, y al igual que a los padres de familia a ellos también se les leyeron las preguntas para que solamente se enfocarán en responder y de esa forma hacer un poco más rápida su participación ya que trabajan en escuelas multigrado (como la Nicolás Bravo de La Mesa).

Nos presentábamos para después comenzar con la lectura, todo se tenía que hacer en un ambiente de respeto. El acercamiento con los docentes era menos que el que lográbamos con algunos padres de familia.

\subsection{Análisis de los instrumentos}

A continuación se presentará el análisis de cada uno de los instrumentos aplicados, dividiéndolos en: historias de vida, observaciones, entrevistas a padres y entrevistas a docentes.

\subsubsection{Historias de vida}

De las 30 historias de vida que se aplicaron, se leyeron meticulosamente cada una para poder obtener categorías y así obtener los resultados requeridos. Los datos obtenidos fueron los siguientes: 


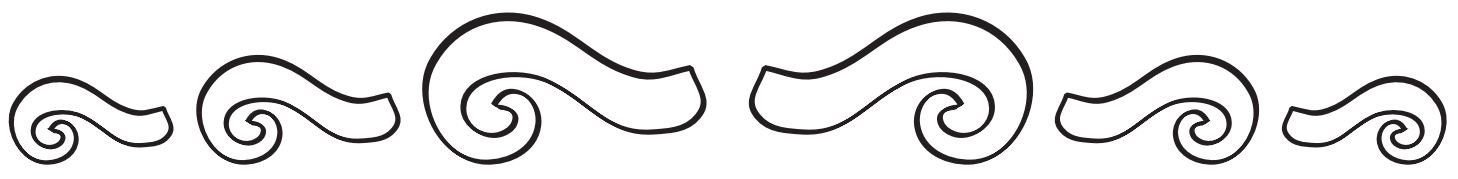

\begin{tabular}{|c|c|}
\hline CATEGORÍAS & DESCRIPCIÓN DE DATOS \\
\hline $\begin{array}{l}\text { Comprensión } \\
\text { lectora }\end{array}$ & $\begin{array}{l}\text { Todos los alumnos mostraron cierta comprensión lectora; ya que } \\
\text { fueron capaces de describir la historia sobre el libro que más les } \\
\text { significó en su vida, aunque la mayoría de ellos escribieron un } \\
\text { resumen sobre la historia, y otros poco fueron capaces de emitir su } \\
\text { propia opinión. } \\
\text { Con relación a las observaciones que se hicieron de ellos con } \\
\text { respecto a la lectura, todos mostraron entender un texto pero a } \\
\text { varios les costaba poder expresar un comentario sobre lo que } \\
\text { pasaba. }\end{array}$ \\
\hline $\begin{array}{l}\text { Apreciación } \\
\text { lectora }\end{array}$ & $\begin{array}{l}\text { Solo } 13 \text { de } 30 \text { alumnos expresaron los sentimientos que obtuvieron } \\
\text { mediante la realización de la lectura. } \\
\text { La mayoría de los niños sólo comentaron sobre la lectura y sobre lo } \\
\text { que trata, pocos fueron los que describieron todos aquellos senti- } \\
\text { mientos que les fueron provocados mediante la lectura. Otros } \\
\text { expresaron lo que la lectura les dejó como enseñanza. } \\
\text { Realizaron una reflexión sobre el texto, y algunos otros cuestiona- } \\
\text { ron el contenido; un chico realizó una crítica al texto. }\end{array}$ \\
\hline $\begin{array}{l}\text { Muestra aplicación } \\
\text { de técnicas vistas en } \\
\text { el taller (etapas de la } \\
\text { lectura: pre - lectura } \\
\text { y pos - lectura) }\end{array}$ & $\begin{array}{l}\text { Algunos chicos contaron que llevan a cabo un proceso durante la } \\
\text { lectura. } \\
\text { Contaron cómo es que seleccionan un libro para leer, todos dijeron } \\
\text { que lo primero que observan es el título y la imagen que tiene la } \\
\text { portada. } \\
\text { Llevan a cabo la poslectura, porque son capaces de escribir lo que } \\
\text { entendieron del tema, y no sólo realizar un resumen. Asumen lo } \\
\text { que pasó en la historia, cómo se sintieron y qué es lo que aprendie- } \\
\text { ron de ello.. }\end{array}$ \\
\hline $\begin{array}{l}\text { Ortografía y } \\
\text { signos de } \\
\text { puntuación. }\end{array}$ & $\begin{array}{l}\text { Todas las historias de vida que los alumnos presentan por lo menos } \\
\text { tienen una falta de ortografía y un máximo de } 11 \text {. En cuanto a los } \\
\text { signos de puntuación, cuatro de ellas no presentan ningún signo. }\end{array}$ \\
\hline $\begin{array}{l}\text { Problemas de } \\
\text { redacción }\end{array}$ & $\begin{array}{l}\text { De las } 30 \text { historias de vida, } 5 \text { historias de vida presentan falta de } \\
\text { congruencia en lo que escriben y no hay ilación de ideas. }\end{array}$ \\
\hline
\end{tabular}




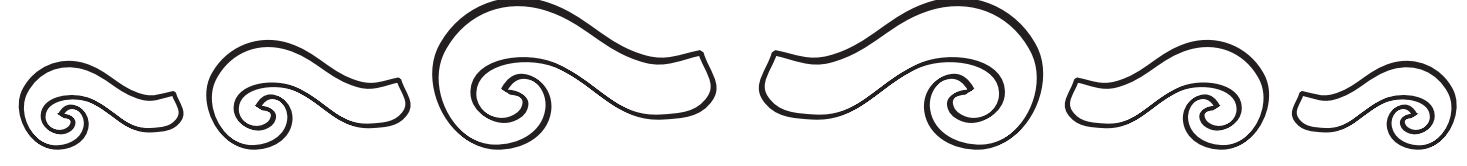

\subsubsection{Observaciones}

De las 30 observaciones realizadas, se obtuvieron los siguientes resultados:

\begin{tabular}{|l|l|}
\hline Acciones a evaluar & Cumple o no descripción de datos \\
\hline $\begin{array}{l}\text { Lee con fluidez un texto de } \\
\text { páginas mínimo }\end{array}$ & $\begin{array}{l}\text { De los } 30 \text { alumnos observados, el } 80 \% \text { de ellos fueron } \\
\text { capaces deleer con fluidez. }\end{array}$ \\
\hline $\begin{array}{l}\text { Al final de la lectura logra } \\
\text { detectar la idea principal }\end{array}$ & $\begin{array}{l}\text { De los } 30 \text { alumnos observados, el } 80 \% \text { fue capaz de } \\
\text { detectarla idea principal. }\end{array}$ \\
\hline $\begin{array}{l}\text { Al final de la lectura es } \\
\text { capaz de resumirla }\end{array}$ & $\begin{array}{l}\text { De los } 30 \text { alumnos observados, 83.3\% fue capaz de } \\
\text { resumirla lectura. }\end{array}$ \\
\hline $\begin{array}{l}\text { Es capaz de entender el } \\
\text { mensaje principal }\end{array}$ & $\begin{array}{l}\text { De los } 30 \text { alumnos observados, el } 83.3 \% \text { fue capaz de } \\
\text { entender elmensaje principal. }\end{array}$ \\
\hline $\begin{array}{l}\text { El alumno emite una } \\
\text { opinión fundamentada } \\
\text { sobrelalectura }\end{array}$ & $\begin{array}{l}\text { De los } 30 \text { alumnos observados, el } 86.6 \% \text { de los alumnos } \\
\text { fueron capaces de dar suopinión. }\end{array}$ \\
\hline
\end{tabular}

\subsubsection{Entrevistas a padres de familia (muestra)}

Se entrevistaron a 25 padres y madres de familia. Se escribirá cada una de las preguntas de la entrevista y las respuestas en porcentaje.

- Pregunta1: Durante la convivencia en el hogar, ¿cuánto tiempo le dedica a la lectura con sus hijos?

Pudimos notar que un $45 \%$ de los encuestados dedica muy poco tiempo con sus hijos para la lectura.

\begin{tabular}{|c|c|c|}
\hline RESPUESTAS & PORCENTAJES & \\
\hline a) $0-15 \mathrm{~min}$ & $45 \%$ & 0-15 min \\
\hline b) $15-30 \mathrm{~min}$ & $18 \%$ & 15-30 min \\
\hline c) $30-60 \mathrm{~min}$ & $18 \%$ & 30-60 min \\
\hline d) Más de $60 \mathrm{~min}$ & $18 \%$ & Mas de $60 \mathrm{~min}$ \\
\hline
\end{tabular}

- Pregunta 2: ¿Ha observado si sus hijos tienen gusto por la lectura?

E1 92\% de los encuestados afirmaron que a sus hijos les gusta leer, esto indica que tal vez los niños sí tengan interés, pero los padres no dedican tiempo para supervisar que leen.

Pudimos notar que un $45 \%$ de los encuestados dedica muy poco tiempo con sus hijos para la lectura.

\begin{tabular}{|l|c|c|}
\hline RESPUESTAS & PORCENTAJES \\
\hline a) Sí & $92 \%$ & \\
\hline c) No & $8 \%$ & \\
\hline
\end{tabular}




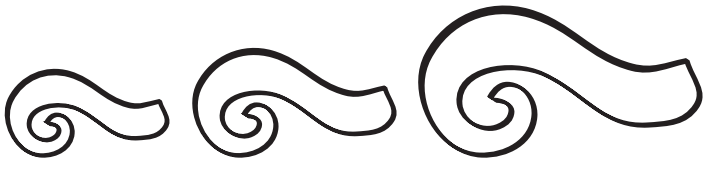

\section{Resultados}

Después de llevar a cabo la investigación y de haber analizado la información que se obtuvo de las encuestas sobre la comprensión lectora; encuestas que se realizaron tanto a padres de familia como a docentes de la comunidad de Ayahualulco, se obtuvieron los siguientes resultados.

La lectoescritura es un proceso de enseñanza aprendizaje en el que se pone énfasis especialmente en el primer ciclo de la educación primaria. En esta etapa los alumnos, por medio de una serie de actividades, desarrollan las capacidades necesarias para poder construir sus ideas y poder interactuar con los otros. Y es que a pesar de que las personas deben aprender a leer y a escribir durante el primer ciclo de la educación, no es así; con respecto a las encuestas realizadas se obtuvo que el $43 \%$ de los padres de familia no saben leer, y por lo tanto no saben escribir.

Como consecuencia de lo anterior es que los padres de familia no ponen la debida atención ni interés sobre la práctica de la lectura con sus hijos; y es que el $45 \%$ de la población de padres solamente dedican a la lectura como máximo 15 minutos. A pesar del alto índice de analfabetismo, se encontró que el $83 \%$ de la población de padres supervisan lo que sus hijos están leyendo. Además el $89 \%$ de los padres se interesan en saber si sus hijos comprenden lo que leen, realizándoles preguntas o pequeñas reflexiones.

Estas reflexiones o preguntas que se les pueden realizar a los niños al terminar alguna lectura son para saber si ellos realmente están comprendiendo. La comprensión lectora no es solo leer, sino que es profundizar también el texto; es la capacidad de poder extraer el sentido de un texto escrito tomando las ideas más importantes y estableciendo vínculos entre ellas. Por lo mismo, los profesores también deben llevar a cabo algún método para darse cuenta si los niños comprenden los textos, con

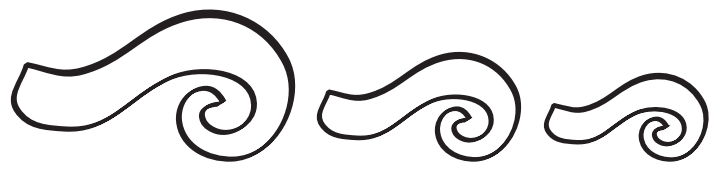

respecto a la encuesta de docentes se obtuvo que todos los maestros (100\%) llevan a cabo alguna actividad para observar si los alumnos comprenden las lecturas.

La lectura es el proceso de recuperación y comprensión de algún tipo de información o ideas almacenadas en un soporte y transmitidas mediante algún tipo de código (Quintero, 2010). Los maestros, para poder llevar a cabo algún tipo de reflexión sobre las lecturas, dedican de 15 minutos a una hora para leer con sus alumnos; en las encuestas se obtuvo que el $50 \%$ lee con sus alumnos de 15 a 30 minutos a la lectura mientras que el otro $50 \%$ le dedica desde 30 minutos a una hora.

Con respecto a las historias de vida que se realizaron a 30 chicos del albergue de La Salle de Ayahualulco, y a las observaciones que se les hicieron a los mismos, descubrimos que el $100 \%$ de los alumnos llegan a comprender un texto, es decir, recuerdan lo que realmente pasó en la historia y quiénes eran los personajes de la misma.

A pesar de que son pocos los chicos que llegan a obtener una reflexión, y demostrar los sentimientos que la lectura les provocó; son capaces de ordenar la historia cronológicamente.

Se pudo observar que los alumnos tienen un cierto acercamiento a la lectura, ya que no lo hacen de manera tan frecuente, ni tampoco tan aislada. Sin embargo, tomando en cuenta la definición a la que llegamos de hábito de lectura: un hábito de lectura es el gusto reiterativo por la práctica de leer constantemente para la recuperación y comprensión de conocimientos, se puede inferir que los alumnos apenas se están acercando a la lectura y que probablemente logren adquirir el hábito de la lectura.

Fue notorio que la ortografía es una deficiencia en ellos y deberán trabajar en ella si quieren mejorar. 


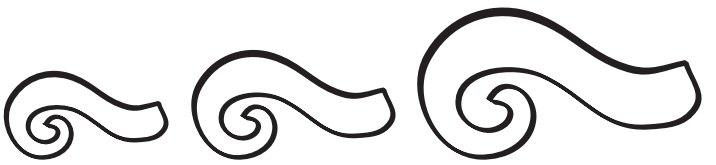

\section{Conclusiones}

\section{1 ¿Cómo se atendió el problema?}

El problema se atendió a través de talleres cuyos propósitos fueron generar el gusto y hábito de los procesos de lectura que se generan en la primaria Vicente Guerrero de la comunidad de Ayahualulco.

La precaria situación económica que padece la comunidad contribuye a que las personas están más interesadas en obtener alimentos y no libros. Solo los profesores realizan un poco de lectura, ya sea sobre literatura, historia, novelas, etc. Tal como se mencionó en el planteamiento del problema; la pobreza y la falta de educación están relacionados y son de los problemas más difíciles que el gobierno mexicano deben enfrentar.

A pesar de que la escuela puede disminuir este problema no lo hace del todo; y es que los profesores no cumplen al $100 \%$ con su trabajo. Los maestros no logran captar la atención de todos los alumnos, o simplemente no cumplen con estar en la escuela cuando se tienen que impartir clases. Los padres de familia van por sus hijos y lamentablemente son los alumnos quienes salen perjudicados.

En los talleres que se proporcionaron dentro de La Salle de Ayahualulco y la primaria de la misma comunidad, se realizaron actividades sobre la lectoescritura con el fin de que los alumnos que recibieran los talleres tomaran más interés sobre ella. Mediante los talleres se observó que los alumnos mostraron interés hacia la lectura. Lo anterior nos da la pauta de las posibles mejoras que se pueden dar con la guía y las actividades correctas.

\subsection{Respuestas a las preguntas de investiga- ción}

En este apartado se dará respuesta a las preguntas que se plantearon al inicio de esta investigación.

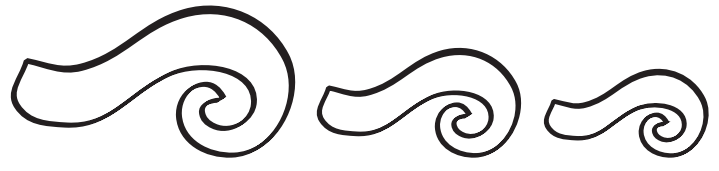

En cuanto a la pregunta 1, la respuesta es que los factores que influyen en el proceso de la lectoescritura son básicamente dos: padres de familia y docentes. Los padres están más preocupados por la situación económica que por proporcionarles libros a sus hijos. Los profesores dejan a los niños a la deriva. Hay otros profesores que sí realizan lecturas dentro del salón, pero no hacen una retroalimentación o platican sobre la lectura que realizan por lo que no llevan un control de lo que los niños aprenden o comprenden.

La situación familiar influye negativamente en el gusto por la lectura por parte de los infantes, quienes al ver que en casa no hay seguimiento o interés por los textos, prefieren ver la televisión o jugar.

En la pregunta 2, las consecuencias son que el nivel académico de la población es muy bajo, muchos alumnos abandonan la escuela y sus aspiraciones académicas son muy cortas.

En la pregunta 3, las debilidades detectadas son la falta del gusto por la lectura, ya que les cuesta mucho trabajo leer, incluso material ajeno a la escuela. Además, pudimos detectar es que los profesores no cumplen al $100 \%$ con su trabajo. Los profesores no logran captar la atención de todos los alumnos, o simplemente no cumplen con estar en la escuela cuando se tienen que impartir clases.

En la pregunta 4, los padres cuentan con una fortaleza: desean que sus hijos tengan mejores niveles académicos que ellos.

La pregunta 5 tendrá respuesta en el apartado de las recomendaciones ya que están ligadas.

\subsection{Cumplimiento de objetivos}

El objetivo general que se planteó al inició de la investigación, no se pudo cumplir completamente; y es que se tuvo el obstáculo de observaciones dentro de la primaria de Ayahualulco. La dinámica dentro de la primaria se dio en 


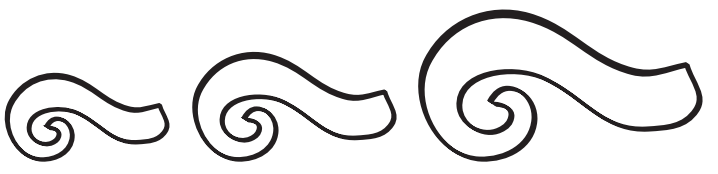

cuatro salones, y durante tres días; en los dos primeros días los chicos de tercer año de primaria no estaban en la escuela, debido a que el profesor de esa aula no se había presentado y los padres de familia fueron por sus hijos. El último día de taller en la primaria se logró trabajar con los chicos de tercer año, pero por la división del equipo de investigación ningún integrante estuvo dentro de esa aula para hacer observaciones.

Aunque gracias a las encuestas que se les realizaron a los docentes de esta primaria se pudo obtener de manera general información sobre cómo trabajan los alumnos de la primaria con respecto a la lectoescritura. El proceso que llevan es distinto dentro del salón de clases, y es que son pocos los profesores que llevan un control sobre lo que sus alumnos están leyendo además de que no se realiza ningún tipo de retroalimentación o ejercicio para saber si los niños comprenden realmente lo que leen.

Los objetivos se alcanzaron en un $90 \%$, ya que uno de los cinco objetivos que se tenían al principio no se pudo cumplir. No se pudo seleccionar a un niño que cursara el tercer año grado en ninguna de las comunidades, y es que todas las escuelas fuera de Ayahualulco eran multigrado además en ellas no se hicieron talleres ni visitas a los chicos, solamente se estuvieron realizando encuestas a los docentes. En la primaria de Ayahualulco tampoco se pudo realizar este objetivo debido a la dificultad presentada anteriormente.

\subsection{Aportaciones del trabajo:}

Con respecto a las situaciones y las cosas que pudimos observar en Ayahualulco lo que la investigación aporta es lo siguiente:

Se elaborará un plan de trabajo con base en las necesidades detectadas de la comunidad lo cual ayudará a disminuir sus debilidades y aumentar sus fortalezas y mantenerlas, con el plan de trabajo se verán beneficiados los alumnos del alberge de los hermanos De LaSalle.

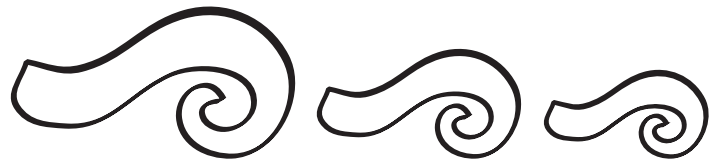

Se atenderían las necesidades y deficiencias que los niños presentan en cuanto a la lectoescritura.

Se darían alternativas y sugerencias que los docentes pueden tomar en cuenta para hacer que los contenidos lleguen como se debe a los alumnos. Esto es con respecto a la escuela de La Salle.

Con respecto a la escuela Vicente Guerrero se elaborará un plan de trabajo en el cual se fomente el gusto por la lectura y se les den estrategias a los docentes para que puedan involucrar a los niños en las reflexiones, actividades para verificar si entendieron el contenido.

Respecto a lo personal, se mostrarán a continuación algunas aportaciones sobre lo que la investigación dejó a cada una de las investigadoras:

- En mi opinión, la investigación fue una experiencia muy enriquecedora ya que no solo aprendí contenidos conceptuales de la materia, sino que también contenidos prácticos al conocer y realizar cada uno de los pasos que se siguen al realizar una investigación cualitativa; además adquirí también contenidos actitudinales ya que se tuvo que trabajar en equipo, adquirí responsabilidades, aprendí a manejar situaciones adversas y a trabajar bajo presión. (Rosa María Romero Martínez).

- Lo que me dejó de experiencia el haber ido a la comunidad de Ayahualulco fue que pude ver la realidad que muchas veces vi por televisión, pero que no había sentido en verdad. Pude ver las condiciones de las personas que ahí viven, me senté a compartir la mesa con ellos. Me di cuenta de que muchas veces nos formamos estereotipos de la gente que vive en comunidades como ésta, y los consideramos flojos e ignorantes, 


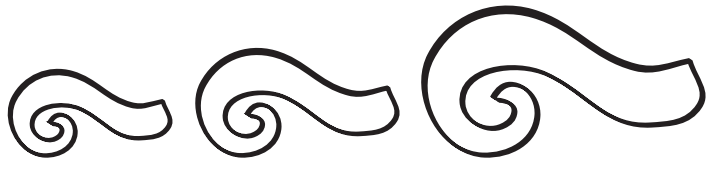

pero la realidad es otra. Hay gente que tiene el deseo de aprender sólo que las personas no cuentan con los recursos para poder hacerlo. Me llevo una gran preocupación por lo que pasa en esa comunidad, un compromiso con los niños del albergue de poder hacer algo que les contribuya a su formación y ¿Por qué no? ver la forma de poder ayudar a las familias que viven en la comunidad de Ayahualulco. (Fiordalizo Maldonado Vargas).

- En general, al finalizar la práctica de la investigación me quedó una gran experiencia; aprendí que no es lo mismo saber que existe la pobreza que verla y vivirla; aprendí que esas familias, a presar de sus carencias, son felices con su familia. Aprendí, además, a valorar lo que tengo en la vida tanto material como emocional, ya que tengo a mi familia y eso es lo más importante para mí.

Con respecto a la carrera, me convencí más de que esto que hago me gusta bastante. Ahora sé qué se siente al estar frente a un grupo y lo difícil que puede ser controlar a un número elevado de alumnos. El estar frente a un grupo es algo increíble porque no solo es dar clases, es también aprender de los alumnos y tenerles un gran aprecio. (Kenneth Gómez Váquez)

- En cuestión individual y en ámbitos emocionales me quedo con el haber valorado lo que poseo, con saber que hay realidades que no podemos ignorar y que podemos hacer mucho por personas que nos necesitan, creo firmemente que después de este proyecto, el día que regresemos lo haremos con mayor conciencia de las necesidades que existen en las comunidades y volveremos con mayores cosas que aportar personal, profesional y emocionalmente. (Paulina Morales Haro).

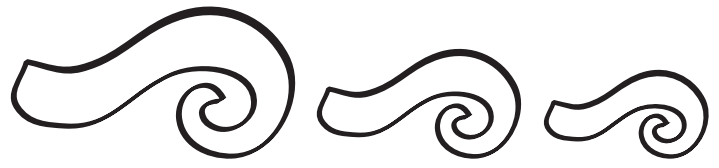

\subsection{Recomendaciones}

Las recomendaciones y estrategias son las siguientes:

- Designar a uno o dos docentes de La Salle Ayahualulco para que vayan a alfabetizar a la población adulta de las comunidades de Ayahualulco.

- Crear un taller sobre lectura de manera permanente tanto en la secundaria como en el bachillerato, en horario de tareas, dos o tres veces por semana. De esta manera se estará desarrollando el hábito por la lectura y rendimiento académico de los alumnos se incrementará de manera positiva. Dentro de este taller, habrá un espacio para la redacción de escritos. Y una estricta vigilancia en las faltas de ortografía con su respectiva corección, esto incrementará la atención por parte de los alumnos en su ortografía y la mejorará.

- Que los maestros de cada asignatura realicen un diagnóstico y una evaluación respecto al nivel de lectoescritura que tienen sus alumnos. A partir de ahí, ellos podrán saber mejor las necesidades que sus estudiantes tienen y podrán abordarlas poco a poco en sus clases y a lo largo del curso.

- Brindar, desde La Salle Ayahualulco, cursos de preparación en la lectoescritura a los docentes de las primarias de las comunidades aledañas. La intención es que ellos puedan transmitir esas habilidades en sus escuelas con sus alumnos.

- Tener como tarea que los alumnos se lleven a casa un libro al mes para leerlo. Esto con el fin de que el hábito de la lectura se vaya fortaleciendo, se familiaricen con otros géneros literarios. Además, que se les requiera un reporte de lectura, en donde se tomará muy en cuenta la ortografía y la expresión escrita. 


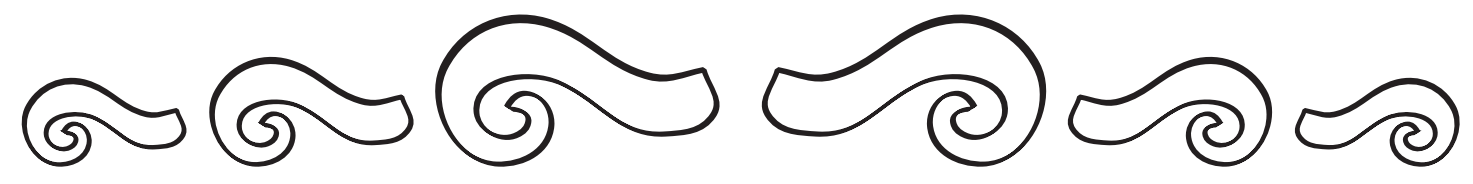

\section{Fuentes de consulta:}

ÁLVAREZ, S. (2004). La lectura. Ecuador: LIBRESA.

CHÁVEZ, S. (2001). La apropiación de la lengua escrita: un proceso constructivo, interactivo y de producción cultural. Consultado el día 04 de septiembre de 2012.

CANU Mirella.(s/f). Lectoescritura:iAprendizaje espontáneo o escolar?. Recuperado de: http://www.lecturayvida.fahce.unlp.edu.ar/numeros/ a15n4/15_04_Canu.pdf. Consultado el día 04 de septiembre de 2012.

DAFONSECA, V. (2010). Dificultades de Aprendizaje. Barcelona: Trillas.

FREEMAN, Y. (1989). Métodos de lectura en español: ireflejan nuestro conocimiento actual del proceso de lectura? Recuperado de: http://www.lecturayvida.fahce.unlp.edu.ar/numeros/ a9n3/09_03_Freeman.pdf. Consultado el día 29 de septiembre de 2012.

IVICH, I. (1987). Lev Semionovich Vygotsky. Teoría del desarrollo mental y problemas en la educación. Recuperado de: http://www.educar.org/articulos/vygotsky.asp. Consultado el día 04 de septiembre de 2012.

KELLY, W. (1982). Psicología de la Educación. España: Morata.

LEÓN, C. (2007). Secuencias de desarrollo infantil integral. Caracas: UCAB

LÓPEZ, M. Ramos, R. Mancilla, M. (2008). La adquisición de la lectoescritura en educación básica desde las teorías interaccionistas sujeto-ambiente. El caso de tres escuelas primarias de Tuxtla Gutiérrez, Chiapas. Consultado el 04 de septiembre de 2012, en: http://www.comie.org.mx/congreso/memoriaelectronica/v10/pdf/area_tematica_01/ponencias/1055-F.pdf

LORENZO, E. (1994). Fundamentos de lingüística. Uruguay: COLIHUE SEPE.

MARIN, O. Zabala, R. Giraldo, S. Cardona, G. (2008). La lectoescritura una estrategia pedagógica para mejorar el rendimiento académico de los estudiantes del grado cuarto de la I.E.R.Pio X del municipio de Ituango. Consultado el 03 de septiembre de 2012 en http://biblioteca.ucn.edu.co/ rdocumentos/tgrados/Documentos/MARIN_Omar_ZABALA_Robinson.pdf

RUIZ, R. (2007). Lectoescritura. Recuperado de: http://rthmruiz8039.wordpress.com Consultado el día 12 de octubre de 2012.

VERNÓN, S. (2011).Tres distintos enfoques en las propuestas de alfabetización inicial. Recuperado de: http://www.dgespe.sep.gob.mx/sites/default/files/pemde/lectura/tdea.pdf. Consultado el día 04 de septiembre de 2012.

ZABALA, A., Alsina, P., Bantula, J., Carranza M., Dilme, D., Forrellad, M., Gratacos, R., Noguerol, A., Oliver, M., Oro, I., Ballonga, P., Rios, J. (2000). Cómo trabajar los contenidos procedimentales en el aula. España: GRAO.

(S/A). (2006). Definiciones. Recuperado de http://www.webdianoia.com. Consultado el día 18 de octubre.

$$
\text { 20) } 43 @
$$




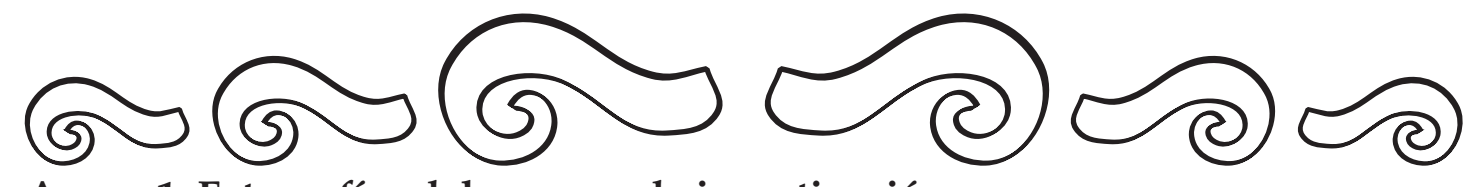

Anexo 1. Fotografías del proceso de investigación

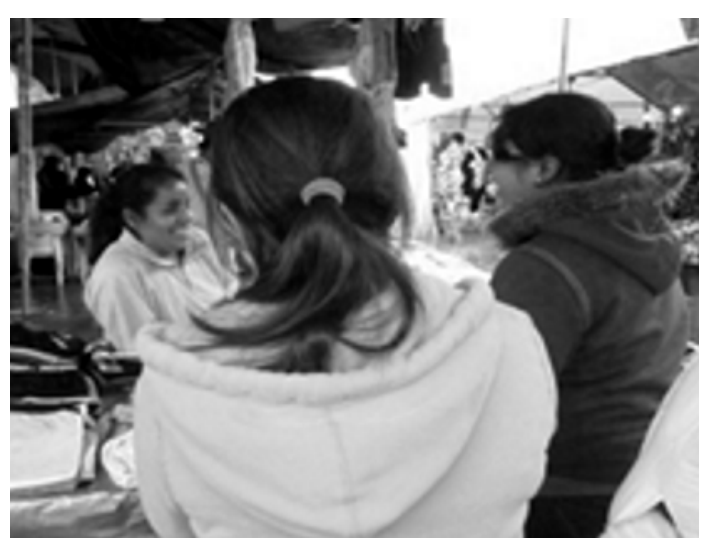

Primeras entrevistas en Coscomatepec

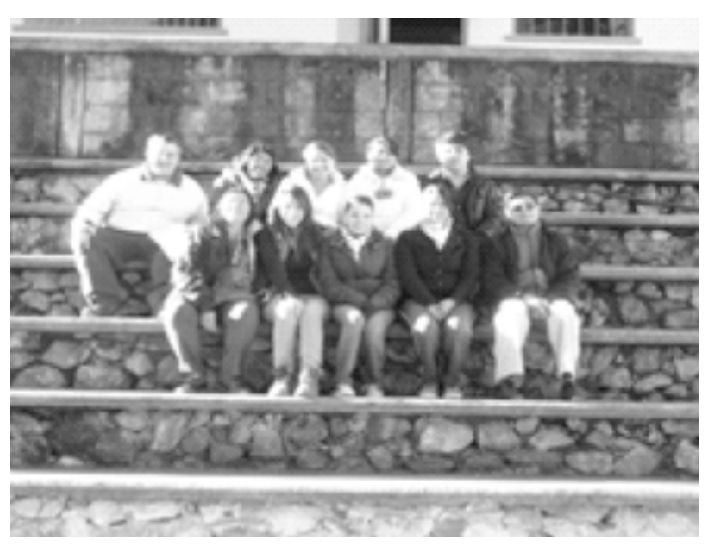

Despedida del albergue de La Salle Ayahualulco

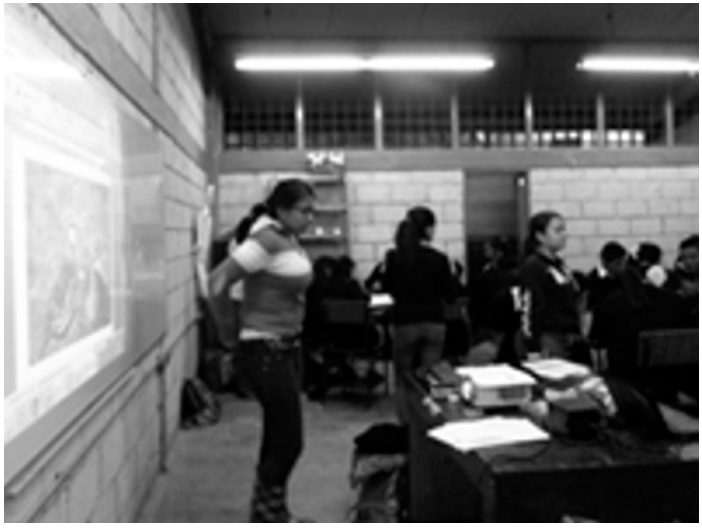

Talleres en el Bachillerato de La Salle Ayahualuco
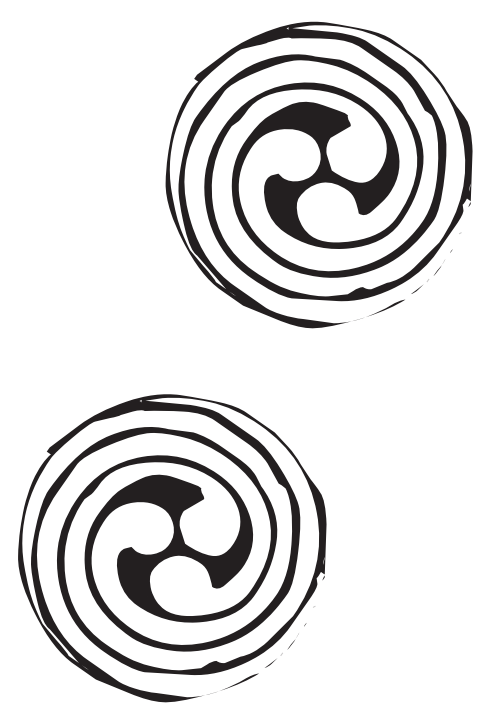
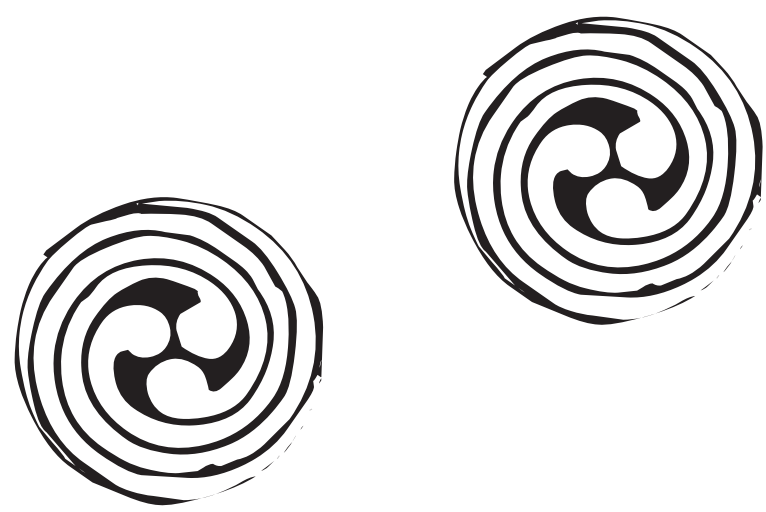